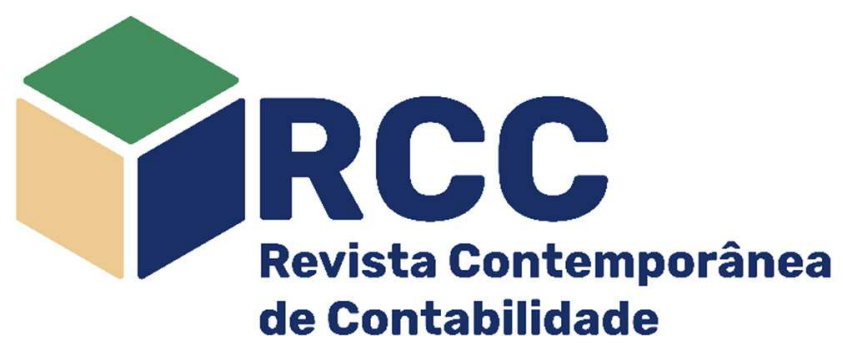

\title{
Conexões sociais e rotatividade involuntária do CEO: evidências do mercado brasileiro
}

\author{
Social connections and involuntary CEO turnover: evidence from the Brazilian market \\ Conexiones sociales y rotación involuntaria del CEO: evidencias del mercado brasileño
}

\author{
Letícia Gomes Locatelli \\ Doutoranda em Ciências Contábeis (UNISINOS), \\ Porto Alegre/RS, Brasil \\ leticiaglocatelli@gmail.com \\ https://orcid.org/0000-0003-3451-2269
}

Fernando Maciel Ramos Doutor em Ciências Contábeis (UNISINOS) Professor do PPG em Administração na Universidade do Contestado, Concórdia/SC, Brasil framos@unc.br https://orcid.org/0000-0002-4222-1253 (0)

Cristiano Machado Costa Doutor em Economia pela University of Pennsylvania Professor do PPG em Ciências Contábeis (UNISINOS), Porto Alegre/RS, Brasil Email: cristianocosta@unisinos.br ORCID: https://orcid.org/0000-0001-9130-2562 (])

Endereço do contato principal para correspondência* Rua Nilo Peçanha 1600, Boa Vista, CEP: 91330-002 - Porto Alegre/RS, Brasil

\section{Resumo}

Este estudo teve como objetivo investigar se as conexões sociais entre os membros do conselho de administração (CA) e o Chief Executive Officer (CEO) diminuem a probabilidade de rotatividade involuntária do CEO no contexto brasileiro. A partir dos dados do Formulário de Referência entre 2012 e 2018 das empresas listadas na Bolsa Brasil Balcão $[B]^{3}$, desenvolveu-se um Índice de Conexão Social (ICS). Os resultados da regressão logística indicam que a probabilidade de rotatividade involuntária do CEO diminui quando o ICS aumenta, consistente com estudos precedentes. O resultado se manteve mesmo na presença de indicadores de governança corporativa e de demais controles. Os resultados evidenciam, assim, que as conexões sociais afetam o monitoramento do CA quanto à rotatividade, bem como que a análise agregada dos dados através do ICS proposto captura melhor este efeito no mercado brasileiro.

Palavras-chave: Conselho de administração; Conexões Sociais; CEO; Rotatividade Involuntária

\begin{abstract}
This study aimed to investigate whether the social connections between the members of the Board of Directors and the Chief Executive Officer (CEO) decreases the probability of involuntary turnover of the CEO in the Brazilian context. We collected data from Formulário de Referência between 2012 and 2018 of the companies listed on the Brasil Bolsa Balcão $[\mathrm{B}]^{3}$ and developed a Social Connection Index (SCl). Logistic regression results indicate that the probability of involuntary turnover of the CEO decreases as the $\mathrm{SCl}$ increases, consistent with the previous studies. Results are significant even in the presence of corporate governance indicators and additional control variables. Results show that the social connections affect the monitoring ability of the Board with respect to the CEO turnover decision. It also shows that the aggregated analysis of the data through the proposed SIC better captures this effect in the Brazilian market.
\end{abstract}

Keywords: Board of Directors; Social Connections; CEO; Involuntary turnover

\section{Resumen}

Este estudio tuvo como objetivo investigar si las conexiones sociales entre los miembros de la Junta Directiva y Chief Executive Officer (CEO) disminuyen la probabilidad de reemplazo involuntario del CEO en el contexto brasileño. A partir de los datos del Formulario de Referência entre 2012 y 2018 de las empresas que cotizan en la Bolsa Brasil Balcão [B] ${ }^{3}$, se desarrolló un Índice de Conexión Social (ICS). Los resultados de la regresión 
logística indican que la probabilidad de reemplazo involuntario del CEO disminuye con las conexiones sociales, consistente con estudios previos. El resultado se mantuvo incluso en presencia de indicadores de gobierno corporativo y características estructurales de la Junta Directiva. Los resultados muestran que las conexiones sociales inciden en el seguimiento de la Junta en términos de facturación, así como el análisis agregado de los datos a través del ICS propuesto capta mejor este efecto, en el mercado nacional.

Palabras clave: Junta de Directores; Conexiones sociales; CEO; Reemplazo involuntario

\section{Introdução}

A separação entre controle e propriedade trouxe à luz questionamentos quanto aos potenciais conflitos advindos da relação entre os proprietários e os gestores. O desalinhamento dos interesses dos atores da relação empresarial pode ocorrer entre o principal e o agente, mas também pode desenvolver-se entre os principais essencialmente na presença de concentração acionária. (Jensen \& Meckling, 1976; Sutton et al., 2018; Young et al., 2008). Neste cenário, os acionistas majoritários exercem seu controle às custas dos acionistas minoritários expropriando-os, visto que não querem arriscar perder o controle da empresa e ocasionando menor proteção legal ao investidor (Harris \& Raviv, 2008; La Porta et al., 1999; Young et al., 2008; Céspedes et al., 2010).

Dentre as práticas de governança corporativa (GC) que se mostram como instrumentos hábeis a mitigar estes conflitos, está a existência de um conselho de administração (CA) independente. A independência dos membros do CA usualmente está associada não existência de relações comerciais e familiares destes com a empresa, não observando-se, proém, aspectos relacionados às experiências pregressas comuns (background educacional, profissional e hobbies, por exemplo) que caracterizam conexões sociais entre membros do CA e o CEO da empresa e podem comprometer a eficiência do CA no papel de monitoramento (Fracassi \& Tate, 2012; Hoitash, 2011; Krishnan et al., 2011; Schmidt, 2015; Westphal, 1999, Hermalin \& Weisbach, 1998).

Dentre as atividades de monitoramento que podem ser afetadas pela presença de conexões sociais está a decisão de demitir ou não um CEO. Pesquisas sinalizam que as conexões sociais entre o CEO e os membros do CA estão associadas à redução da rotatividade involuntária do CEO (Balsam et al, 2017; Hwang \& Kim, 2009). Assim, em um mercado emergente e caracterizado pela concentração acionária como o Brasil, torna-se relevante investigar a influência das conexões sociais entre CEO e os membros do CA especialmente quanto à troca de CEO. Assim, o objetivo do estudo é investigar se as conexões sociais entre os membros do CA e o CEO diminuem a probabilidade de rotatividade involuntária do CEO no contexto brasileiro.

A literatura acerca deste tema é exígua. Não identificaram-se estudos precedentes que analisem a influência das conexões sociais na rotatividade involuntária do CEO para o mercado brasileiro, consistindo, portanto, em uma lacuna de pesquisa a ser preenchida. Da mesma forma, desconhece-se a existência de estudos que abordem a perspectiva das conexões sociais a partir de um índice único. Identificam-se estudos que analisam os impactos das conexões sociais a partir da perspectiva de análise de redes sociais a exemplo dos estudos de Liu (2014), Renneboog e Zhao (2017) e Gao et al. (2017).

Os resultados deste estudo fornecem evidências empíricas sobre o mercado brasileiro indicando uma proporção maior de empresas onde o CEO e os membros do CA estão conectados socialmente em comparação a outros mercados. Indicam, ainda, que a probabilidade de rotatividade involuntária do CEO é menor em relação a um CEO sem conexões sociais mesmo na presença de controles relacionados a mecanismos de GC, corroborando estudos anteriores desenvolvidos em outros mercados (Balsam et al., 2017; Hwang \& Kim, 2009) .

Além disso, o estudo propõe desenvolver um Índice de Conexão Social (ICS), que mensura o nível de conectividade do CEO com os membros do CA, contribuindo para a compreensão pelos stakeholders sobre a existência e mensuração das conexões sociais. Permite, ainda, a comparabilidade dos efetios das conexões sociais entre empresas a partir de um índice único que demonstra o quão conectado socialmente estão o CEO e o CA. Neste sentido, a pesquisa possui uma contribuição metodológica, pois avança em relação às pesquisas precedentes, as quais avaliaram a conexão social a partir de indicadores isolados ou sob a perspectiva da análise de redes sociais.

A pesquisa está estruturada em seis seções sendo a primeira a introdução, seguida do referencial teórico. Após, é apresentada a metodologia da pesquisa e na quarta seção os resultados obtidos. Estes são discutidos na quinta seção, e, por fim, as considerações finais.

\section{Referencial Teórico}

\subsection{Conselho de administração e conexões sociais}

O CA é um mecanismo de ligação entre acionistas e gestores com relevância na estrutura de governança das organizações (Jensen, 1993). Os manuais de boas práticas de governança corporativa recomendam que o CA deve ser composto, preferencialmente, por membros externos e declarados 
independentes (Brasil, Bolsa, Balcão, 2018; Cadbury, 1992; Interagentes, 2016; IBGC, 2015; OECD, 2016). A definição formal de independência está usualmente atrelada à ausência de relações familiares, financeiras ou corporativas entre os membros do CA e o CEO ou entre aqueles e a empresa. Desconsiderando, portanto, as conexões advindas de backgrounds passados como, por exemplo, quando o CEO e os membros do CA compartilham experiências anteriores por meio de instituições de ensino, atividades profissionais, clubes sociais, etc. (Hwang \& Kim, 2009; Krishnan et al., 2011).

As relações mediadas pela existência de conexões sociais podem proporcionar um sentimento de pertencimento que, por consequência, pode facilitar a interação pessoal através da homofilia (afinidade por pessoas similares); a qual prediz que as pessoas se mostram mais confortáveis com aquelas com quem compartilham características e semelhanças (McPherson et al., 2001). A literatura relaciona efeitos ambíguos para a empresa quando este sentimento é compartilhado entre CEO e membros do CA. Se por um lado favorece a troca de informação melhorando o envolvimento do CA nas decisões estratégicas (Adams \& Ferreira, 2007; Hoitash, 2011), por outro, pode implicar em um baixo monitoramento por parte do CA com o favorecimento de decisões que benficiem o CEO (Schmidt, 2015).

Os resultados do estudo de Hwang e Kim (2009) apontam que CAs socialmente independentes concedem um nível significativamente mais baixo de remuneração e apresentam maior sensibilidade de rotatividade ao desempenho do que empresas cujos CA foram classificados tão somente como formalmente independentes (ausência de relação comercial com a empresa ou com o CEO, ausência de relação familiar com os diretores da empresa e ausência de interlocking). Hoitash (2011) indica que a presença de conexões sociais está associada a uma maior remuneração dos gestores enquanto que Krishnan et al (2011) indicam uma associação entre as conexões sociais e o gerenciamento de resultados.

Sob este enfoque, a independência formal desprovida de independência social pode ser insuficiente para assegurar a eficácia dos conselhos ao executar sua função de monitoramento (Tung, 2011). Dentre as funções de monitoramento exercidas pelo CA que podem ser afetadas pela existência de conexões sociais está a rotatividade involuntária do CEO, a qual pode se tornar mais difícil no caso de haver ligação entre o CEO e os membros do CA como tratado na próxima seção.

\subsection{Conexões sociais e rotatividade involuntária do CEO: estudos precedentes e desenvolvimento da hipótese}

O CA possui dentre o seu escopo de decisões a contratação e demissão do CEO, sendo essa última caracterizada como rotatividade involuntária. A rotatividade involuntária do CEO pode estar relacionada com diferentes questões que podem ser classificadas em aspectos gerenciais (Kaplan \& Minton, 2012; Gao et al., 2017; Hu \& Kim, 2019), características próprias do cargo (Brookman \& Thistle, 2009; Bushman et al, 2010) ou pessoais do CEO (Campbell et al., 2011; Gao et al., 2017; Liu, 2014). Neste sentido, uma maior capacidade do CA de avaliar com precisão o desempenho do CEO reduzirá a probabilidade de um erro oneroso, como a retenção de um mau CEO ou a substituição de um bom. (Parrino, 1997).

Entretanto, dado que o CA é composto por pessoas com condições e interesses diversos (financeiros, participação acionária, vinculação com acionistas e/ou controladores, etc), seus membros terão incentivos pessoais diferentes para executar suas funções. Da mesma forma, os membros do CA também se diferem em termos de relacionamento com o CEO e disposição de monitorá-lo. Assim, a independência do CA será relevante, pois presume-se que membros do CA mais independentes estejam mais dispostos a fazer esforços para monitorar o CEO do que membros não independentes (Hermalin \& Weisbach, 1998; Knyazeva et al, 2013).

Neste contexto, as conexões sociais dos membros do CA com o CEO assumem importância, pois influenciam nos incentivos e disposição do CA de monitorar o CEO. Guo e Masulis (2015) indicam que em conselhos menos independentes é necessário um declínio maior no desempenho da empresa para diminuir a avaliação do CEO dado que conselhos mais independentes são mais dispostos a substituir o CEO do que CAs não-independentes. Estudos empíricos relacionam a presença de CEOs duais e de conexões sociais ou de relações comerciais entre os membros do CA e o CEO negativamente à probabilidade de rotatividade do CEO.

Hwang e Kim (2009) ao analisarem uma amostra norte americana, verificaram a alteração do monitoramento do CA quando este é classificado como convencional ou socialmente independente. As evidências apontaram que as empresas com conselhos socialmente independentes possuem maior sensibilidade à rotatividade por desempenho do que aquelas em que o conselho é apenas formalmente independente. Coles e Naveen (2014) relacionam o fato dos conselheiros serem indicados após a posse do CEO (cooptação) com o baixo monitoramento. Suas evidências demonstraram que a medida que a cooptação aumenta, a sensibilidade à rotatividade diminui. Balsam et al. (2017) decompuseram a rotatividade em voluntária e involuntária e as evidências obtidas para empresas dos Estados Unidos demonstraram associação entre a existência de conexões sociais entre o CEO e os membros do CA à redução da probabilidade de rotatividade involuntária do CEO.

A mitigação do monitoramento na presença de conexões sociais pode ser acentuada em mercados de concentração acionária onde, em casos extremos, os acionistas controladores podem determinar a 
composição de todo o CA anulando sua capacidade de monitoramento (Balsam et al., 2017). O estudo de Vieira e Martins (2018) corrobora esta afirmativa ao evidenciar que a concentração acionária diminui a sensibilidade à rotatividade do $\mathrm{CEO}$ no contexto brasileiro. O Brasil é um mercado emergente onde a maioria das companhias possui elevado nível de concentração acionária, (Lana et al., 2018). Cabe destacar que na amostra analisada neste estudo a média de ações detidas pelo primeiro acionista é de $49,80 \%$. Neste cenário, aas conexões sociais podem ser determinantes para a eficiência do CA.

Desta forma, entende-se que as conexões sociais havidas entre os membros do CA e o CEO podem interferir nas decisões estratégicas tomadas pelo conselho, essencialmente quanto à contratação ou demissão do CEO. Um CA conectado socialmente ao CEO pode ser mais resistente a demiti-lo do que um CA socialmente independente. Espera-se, então, uma relação negativa entre a existência de conexão social entre os membros do CA e o CEO e a probabilidade de rotatividade involuntária do CEO, o que enseja a seguinte hipótese de pesquisa:

H: A existência de conexões sociais entre os membros do CA e o CEO diminui a probabilidade de rotatividade involuntária do CEO. seção.

A fim de testar a hipótese apresentada nesta seção, o percurso metodológico é descrito na próxima

\section{Metodologia}

\subsection{População e amostra}

A população do estudo compreende 364 empresas de capital aberto listadas na $[\mathrm{B}]^{3}$ entre os anos de 2012 a 2018. Foram excluídas da amostra as empresas financeiras em virtude das características peculiares que podem desviar os resultados. Também foram excluídas empresas com apenas um ano de informação dada a impossibilidade de identificação de rotatividade involuntária de CEO e uma empresa que apresentou dois CEOs no mesmo ano por inviabilizar a apuração de um único Índice de Conexão Social (ICS).

Dado que o ICS é estruturado pelo cruzamento de dados do CEO e dos membros do CA, elegeu-se como dado de maior pertinência a Instituição de Ensino Superior (IES) de formação do CEO excluindo-se da amostra as empresas para as quais não se tinha a informação de IES do CEO. Evidencia-se na Tabela 1 a composição da amostra final.

\section{Tabela 1:}

\section{Composição da Amostra}

População 364

(-) Setor Financeiro e similares

(-) Empresas com 1 ano de informação

(-) Empresa com 2 CEOs
(-) Empresas sem dados de IES do CEO

Amostra final

Fonte: Dados da pesquisa (2020).

A partir da disponbilidade de dados, obteve-se uma amostra desbalanceada de 199 empresas que culminou em 959 observações.

\subsection{Operacionalização da pesquisa}

A pesquisa emprega como técnica de análise multivariada a regressão logística tendo em vista que as variáveis de resposta utilizadas neste estudo são binárias (Gujarati \& Porter, 2011). Foi utilizado o método de estimação por máxima verossimilhança através de uma função logística com pool de cross-sections com clusterização dos resíduos por empresa para se estimar a probabilidade de ocorrência do evento com a equação 1.

$$
P_{j} \quad\left(y_{i t} \neq 0 \mid x_{i t}\right)=e^{\varepsilon_{i t}} /\left(1+e^{\varepsilon_{i t}}\right)
$$

onde $\varepsilon$ é estimado da através da equação 2

$$
\operatorname{ROTINV}_{i t}=\beta_{0}+\beta_{1} \text { ICS }_{\text {it }-1}+\sum_{k=1}^{m}\left\{\gamma_{k} \text { Controles }_{k i t-1}\right\}+\varepsilon_{i t}
$$

A variável dependente rotatividade involuntária $\left(R O T I N V_{i t}\right)$ é uma variável binária que identifica se o CEO foi demitido (1) ou não (0). Considera-se rotatividade involuntária se na data da saída $(t)$ o CEO tem menos de 60 anos e este não se mantém ou não se recoloca no mercado em cargo de CEO, no CA ou em Diretoria Executiva da mesma empresa ou de outra empresa listada na $[B]^{3}$, em até dois anos de sua saída (Balsam et al., 2017; Coles et al., 2014; Hwang \& Kim, 2009). Adicionalmente, classificou-se como rotatividade voluntária $\left(R O T V O L_{i t}\right)$ se o CEO se mantém ou se recoloca no mercado em até 2 anos da sua saída, ou ainda, se possui mais de 60 anos no momento da saída $(t)$. 
A variável independente $\left(I C S_{i t-1}\right)$ é definida conforme equação 3 . O ICS consiste em uma escala que varia entre 0 e 1 . Assim, um ICS de 0 indica que nenhum membro do CA possui conexão com o CEO enquanto um ICS de 1 indica que todos os membros do CA são conectados com o CEO.

O ICS foi construído a partir de 5 indicadores que representam fontes de conexão social já apontadas pela literatura, a saber: i) relação familiar, ii) instituição de ensino superior, iii) experiência profissional, iv) participação em conselhos e v) dualidade do conselheiro. (Chahine \& Goergen, 2013, 2014; Fracassi \& Tate, 2012; Hoitash, 2011; Ishii \& Xuan, 2014; McGuinness, 2018, Balsam et al., 2017; Hwang \& Kim, 2009; Cohen et al., 2010;; Kang et al., 2018; Khedmati et al., 2020; Nguyen, 2012; Young et al., 2008). Os indicadores foram mensurados conforme Apêndice A. O ICS foi operacionalizado através da seguinte equação:

$$
I C S_{i t}=\frac{\sum I N D_{i t}}{5}
$$

onde:

ICS = Índice de Conexão Social do CEO com os membros do CA da empresa $i$ no período $t$.

IND = Indicadores de conexão social do CEO com os membros do CA da empresa $i$ no período $t$.

$5=$ Número de indicadores.

A exemplo do que ocorre na literatura para construção de índices de governança corporativa (Black et al., 2017; Crisóstomo et al., 2020), o índice proposto é operacionalizado pelo somatório simples dos indicadores dividido pelo número de indicadores. Não há ponderação entre os indicadores visto não identificar-se base teórica para atribuir pesos diferentes aos indicadores nesse fenômeno. Estudos anteriores argumentam que índices construídos sem ponderação são menos suscetíveis à subjetividade dos pesquisadores o que facilita sua reprodução (Beiner et al., 2006; Brown \& Caylor, 2004; Black et al., 2017).

O conjunto destes indicadores contempla pontos de interação entre o CEO e os membros do CA já sinalizados pela literatura. Contudo, o efeito de sua análise conjunta se apresenta como uma lacuna que a presente pesquisa se propõe a preencher a partir do desenvolvimento do ICS. Para validação do índice utilizou-se da Técnica Delphi com um painel de seis especialistas, cujos resultados encontram-se no Apêndice B (Cooper \& Schindler, 2016; Martins, 2006, Campbell et al., 2002).

A partir dos estudos anteriores acerca da rotatividade do CEO foram definidas as variáveis de controle (Apêndice A), as quais podem ser classificadas em financeiras, governança, características da empresa, do CEO e do CA.

Os dados referentes às informações pessoais do CEO e dos membros do $C A$, remuneração do CEO, tamanho do conselho, dualidade do CEO, quantidade de mandatos do CEO, independência do CA e nível de governança corporativa foram extraídos do Formulário de Referência entregue pelas empresas listadas na $[B]^{3}$ nos anos de 2011 a 2018. Os dados do Formulário de Referência foram extraídos do site da $[B]^{3}$ através do software estatístico RStudio com a utilização do pacote GetDFPData (Perlin et al., 2018). Dados referentes às características da empresa que configuram variáveis de controle foram obtidos da Economática ${ }^{\circledR}$.

As variáveis foram agrupadas em planilha eletrônica e, posteriormente, exportadas para o software estatístico Stata ${ }^{\circledR}$ onde foram realizadas as análises. As variáveis financeiras foram winsorizadas com limite inferior (1\%) e limite superior (99\%) minimizando os efeitos de outliers, conforme Balsam et al. (2017). Os resultados obtidos são apresentados na seção subsequente.

\section{Estatísticas Descritivas e Resultados}

Os dados indicam que em $19,29 \%$ das observações da amostra houve rotatividade de CEO, destas, $7,71 \%$ foram classificadas como involuntárias (ROTINV). Este percentual é superior ao encontrado por Balsam et al. (2017), que foi de 3,9\%, indicando que as empresas brasileiras tem maior propensão à rotatividade involuntária do que as empresas americanas. Esta diferença pode estar relacionada a maior volatilidade dos ciclos de negócios nos mercados emergentes, ao qual o Brasil está inserido, tornando pertinente a análise desse tema no Brasil.

\section{Tabela 2.}

A estatística descritiva da variável independente e das variáveis de controle estão sintetizadas na

Tabela 2:

\section{Estatística descritiva}

\begin{tabular}{|c|c|c|c|c|c|}
\hline Variável & Observações & Média & Desvio Padrão & Mínimo & Máximo \\
\hline ROTINV & 959 & 0,0771 & 0,2669 & 0 & 1 \\
\hline ICS & 959 & 0,192 & 0,123 & 0 & 0,60 \\
\hline \multicolumn{6}{|c|}{ Características financeiras } \\
\hline Endividamento & 959 & 0,6388 & 0,3498 & 0,1175 & 2,777 \\
\hline$E B I T$ & 959 & 756.168 & 2.315 .748 & -2.128 .620 & 17.572 .598 \\
\hline Tamanho & 959 & 15,0100 & 1,6580 & 10,94 & 19,3 \\
\hline$R O A$ & 959 & 0,0229 & 0,1030 & $-0,441$ & 0,295 \\
\hline Crescimento & 959 & 1,1220 & 0,4320 & 0 & 4,145 \\
\hline Perda & 959 & 0,2980 & 0,4580 & 0 & 1 \\
\hline
\end{tabular}




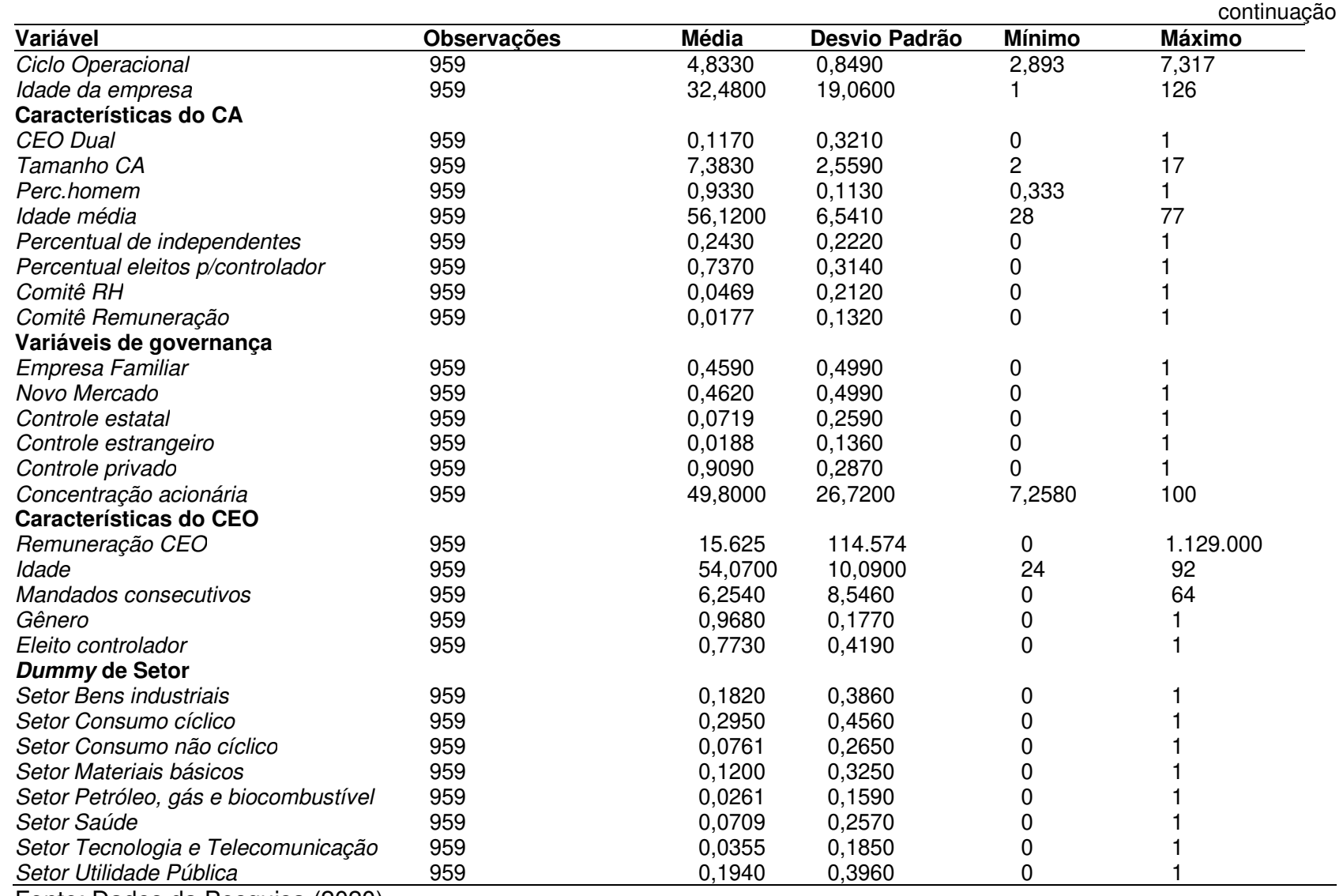

Fonte: Dados da Pesquisa (2020).

$\mathrm{Na}$ amostra analisada, 93,22\% das observações apresentam algum grau de conexão entre o CA e o CEO (ou seja, ICS>0), sendo superior ao achado de Balsam et al. (2017) onde $42,6 \%$ das empresas apresentaram alguma conexão entre CEO e os membros do CA e de Hwang e Kim (2009), onde o percentual observado foi de $37,6 \%$ dos CAs como socialmente conectados. Esse resultando indica uma maior presença de conexão social entre membros do CA e CEOs no Brasil do que nos EUA. Apesar da existência de muitas empresas com conexões entre membros do CA e CEOs relativamente aos EUA, o nível de conexões observado (ICS médio de 0,192) indica um baixo grau de conexão entre os CAs e os CEOs para amostra analisada.

Os resultados das estimativas sobre efeitos das conexões sociais sobre a rotatividade involuntária encontram-se descritos na Tabela 3. As variáveis de controle foram inseridas gradativamente sendo estimadas seis especificações.

Tabela 3:

Resultados para rotatividade involuntária

\begin{tabular}{|c|c|c|c|c|c|c|}
\hline Variáveis & $\begin{array}{l}\text { (1) } \\
\text { ROTINV }\end{array}$ & $\begin{array}{l}\text { (2) } \\
\text { ROTINV }\end{array}$ & $\begin{array}{l}\text { (3) } \\
\text { ROTINV }\end{array}$ & $\begin{array}{l}\text { (4) } \\
\text { ROTINV }\end{array}$ & $\begin{array}{l}\text { (5) } \\
\text { ROTINV }\end{array}$ & $\begin{array}{l}\text { (6) } \\
\text { ROTINV }\end{array}$ \\
\hline$I C S$ & $\begin{array}{l}-4,5696^{\star \star \star} \\
(1,2024)\end{array}$ & $\begin{array}{l}-4,0540^{\star \star *} \\
(1,1681)\end{array}$ & $\begin{array}{l}-4,0247^{\star * *} \\
(1,1410)\end{array}$ & $\begin{array}{l}-3,5493^{* * *} \\
(1,1275)\end{array}$ & $\begin{array}{l}-2,8182^{\star *} \\
(1,1864)\end{array}$ & $\begin{array}{l}-3,2198^{\star \star \star} \\
(1,2385)\end{array}$ \\
\hline Endividamento & & $\begin{array}{l}-0,1153 \\
(0,4012)\end{array}$ & $\begin{array}{l}0,0311 \\
(0,4655)\end{array}$ & $\begin{array}{l}0,0174 \\
(0,4490)\end{array}$ & $\begin{array}{l}0,0839 \\
(0,4459)\end{array}$ & $\begin{array}{l}-0,0872 \\
(0,4685)\end{array}$ \\
\hline$E B I T$ & & $\begin{array}{l}-0,0000^{*} \\
(0,0000)\end{array}$ & $\begin{array}{l}-0,0000^{* *} \\
(0,0000)\end{array}$ & $\begin{array}{l}-0,0000^{\star *} \\
(0,0000)\end{array}$ & $\begin{array}{l}-0,0000^{* *} \\
(0,0000)\end{array}$ & $\begin{array}{l}-0,0000^{* *} \\
(0,0000)\end{array}$ \\
\hline Tamanho & & $\begin{array}{l}0,2264^{* * *} \\
(0,0809)\end{array}$ & $\begin{array}{l}0,1782^{*} \\
(0,1024)\end{array}$ & $\begin{array}{l}0,1525 \\
(0,1059)\end{array}$ & $\begin{array}{l}0,1859^{*} \\
(0,1094)\end{array}$ & $\begin{array}{l}0,2877^{\star *} \\
(0,1251)\end{array}$ \\
\hline$R O A$ & & $\begin{array}{l}0,3354 \\
(1,3831)\end{array}$ & $\begin{array}{l}0,3613 \\
(1,4710)\end{array}$ & $\begin{array}{l}0,6560 \\
(1,6402)\end{array}$ & $\begin{array}{l}1,0424 \\
(1,7119)\end{array}$ & $\begin{array}{l}0,6462 \\
(1,6066)\end{array}$ \\
\hline Crescimento & & $\begin{array}{l}0,3873^{\star * *} \\
(0,1446)\end{array}$ & $\begin{array}{l}0,3710^{\star \star *} \\
(0,1357)\end{array}$ & $\begin{array}{l}0,4223^{\star \star \star} \\
(0,1308)\end{array}$ & $\begin{array}{l}0,3686^{\star * *} \\
(0,1390)\end{array}$ & $\begin{array}{l}0,4889^{\star * *} \\
(0,1592)\end{array}$ \\
\hline Perda & & $\begin{array}{l}1,1375^{\star * *} \\
(0,3435)\end{array}$ & $\begin{array}{l}1,1722^{\star * *} \\
(0,3442)\end{array}$ & $\begin{array}{l}1,2316^{\star \star *} \\
(0,3526)\end{array}$ & $\begin{array}{l}1,2361^{* * *} \\
(0,3571)\end{array}$ & $\begin{array}{l}1,4294^{* * *} \\
(0,4293)\end{array}$ \\
\hline Ciclo Operacional & & $\begin{array}{l}0,0428 \\
(0,1544)\end{array}$ & $\begin{array}{l}0,1290 \\
(0,1558)\end{array}$ & $\begin{array}{l}0,1368 \\
(0,1477)\end{array}$ & $\begin{array}{l}0,1233 \\
(0,1499)\end{array}$ & $\begin{array}{l}0,2235 \\
(0,1719)\end{array}$ \\
\hline Idade da empresa & & $\begin{array}{l}-0,0116^{*} \\
(0,0067)\end{array}$ & $\begin{array}{l}-0,0128^{*} \\
(0,0068)\end{array}$ & $\begin{array}{l}-0,0091 \\
(0,0069)\end{array}$ & $\begin{array}{l}-0,0050 \\
(0,0070)\end{array}$ & $\begin{array}{l}-0,0078 \\
(0,0085)\end{array}$ \\
\hline
\end{tabular}




\begin{tabular}{|c|c|c|c|c|c|c|}
\hline Variáveis & $\begin{array}{l}\text { (1) } \\
\text { ROTINV }\end{array}$ & $\begin{array}{l}\text { (2) } \\
\text { ROTINV }\end{array}$ & $\begin{array}{l}\text { (3) } \\
\text { ROTINV }\end{array}$ & $\begin{array}{l}\text { (4) } \\
\text { ROTINV }\end{array}$ & $\begin{array}{l}\text { (5) } \\
\text { ROTINV }\end{array}$ & $\begin{array}{l}\text { (6) } \\
\text { ROTINV }\end{array}$ \\
\hline CEO Dual & & & $\begin{array}{l}-1,3006 \\
(0,8150)\end{array}$ & $\begin{array}{l}-1,1179 \\
(0,8371)\end{array}$ & $\begin{array}{l}-0,7616 \\
(0,8380)\end{array}$ & $\begin{array}{l}-0,6919 \\
(0,7604)\end{array}$ \\
\hline Tamanho CA & & & $\begin{array}{l}0,0401 \\
(0,0850)\end{array}$ & $\begin{array}{l}0,0491 \\
(0,0835)\end{array}$ & $\begin{array}{l}0,0419 \\
(0,0799)\end{array}$ & $\begin{array}{l}0,0503 \\
(0,0837)\end{array}$ \\
\hline Perc.homem & & & $\begin{array}{l}-0,0108 \\
(1,4742)\end{array}$ & $\begin{array}{l}-0,3226 \\
(1,4620)\end{array}$ & $\begin{array}{l}-0,8547 \\
(1,4619)\end{array}$ & $\begin{array}{l}-0,3069 \\
(1,5424)\end{array}$ \\
\hline Idade média & & & $\begin{array}{l}0,0052 \\
(0,0235)\end{array}$ & $\begin{array}{l}0,0153 \\
(0,0248)\end{array}$ & $\begin{array}{l}0,0225 \\
(0,0240)\end{array}$ & $\begin{array}{l}0,0093 \\
(0,0265)\end{array}$ \\
\hline Perc.independentes & & & $\begin{array}{l}-1,0128^{*} \\
(0,6122)\end{array}$ & $\begin{array}{l}-0,5082 \\
(0,7234)\end{array}$ & $\begin{array}{l}-0,7633 \\
(0,7116)\end{array}$ & $\begin{array}{l}-1,3888 \\
(0,8634)\end{array}$ \\
\hline $\begin{array}{l}\text { Perc. eleitos } \\
\text { p/controlador }\end{array}$ & & & $\begin{array}{l}0,1442 \\
(0,5090)\end{array}$ & $\begin{array}{l}0,4861 \\
(0,6336)\end{array}$ & $\begin{array}{l}0,3998 \\
(0,6412)\end{array}$ & $\begin{array}{l}0,5044 \\
(0,7260)\end{array}$ \\
\hline Comitê $R H$ & & & $\begin{array}{l}0,2333 \\
(0,6445)\end{array}$ & $\begin{array}{l}0,3660 \\
(0,6642)\end{array}$ & $\begin{array}{l}0,2969 \\
(0,6724)\end{array}$ & $\begin{array}{l}0,6663 \\
(0,6459)\end{array}$ \\
\hline Comitê & & & $-0,5526$ & $-0,6870$ & $-0,8055$ & $-1,5500$ \\
\hline Remuneração & & & $(1,2429)$ & $(1,2793)$ & $(1,2824)$ & $(1,4291)$ \\
\hline Concentração & & & & $-0,0021$ & $-0,0004$ & $-0,0007$ \\
\hline acionária & & & & $(0,0067)$ & $(0,0067)$ & $(0,0068)$ \\
\hline Empresa Familiar & & & & $\begin{array}{l}-0,6722^{*} \\
(0,3659)\end{array}$ & $\begin{array}{l}-0,7901^{\star *} \\
(0,3750)\end{array}$ & $\begin{array}{l}-0,4714 \\
(0,3994)\end{array}$ \\
\hline Novo Mercado & & & & $\begin{array}{l}-0,2093 \\
(0,3478)\end{array}$ & $\begin{array}{l}0,0068 \\
(0,3232)\end{array}$ & $\begin{array}{l}-0,0401 \\
(0,3570)\end{array}$ \\
\hline Controle estatal & & & & $\begin{array}{l}-0,7562 \\
(0,6286)\end{array}$ & $\begin{array}{l}-0,5663 \\
(0,6342)\end{array}$ & $\begin{array}{l}-1,1000^{*} \\
(0,6416)\end{array}$ \\
\hline Controle estrangeiro & & & & $\begin{array}{l}1,4678^{* *} \\
(0,7173)\end{array}$ & $\begin{array}{l}1,3058^{*} \\
(0,6812)\end{array}$ & $\begin{array}{l}1,6959^{\star *} \\
(0,8099)\end{array}$ \\
\hline Remuneração CEO & & & & & $\begin{array}{l}0,000{ }^{*} \\
(0,0000)\end{array}$ & $\begin{array}{l}0,0000^{\star *} \\
(0,0000)\end{array}$ \\
\hline Idade & & & & & $\begin{array}{l}-0,0441^{\star * *} \\
(0,0157)\end{array}$ & $\begin{array}{l}-0,0488^{* *} \\
(0,0177)\end{array}$ \\
\hline Gênero & & & & & $\begin{array}{l}0,5473 \\
(0,6089)\end{array}$ & $\begin{array}{l}0,6969 \\
(0,7770)\end{array}$ \\
\hline Eleito controlador & & & & & $\begin{array}{l}-0,0350 \\
(0,3143)\end{array}$ & $\begin{array}{l}-0,1942 \\
(0,3449)\end{array}$ \\
\hline Mandados & & & & & $-0,0166$ & $-0,0060$ \\
\hline consecutivos & & & & & $(0,0242)$ & $(0,0251)$ \\
\hline D.ano & Não & Não & Não & Não & Não & Sim \\
\hline D.Setor & Não & Não & Não & Não & Não & Sim \\
\hline Constante & $\begin{array}{l}-1,7305^{\star \star *} \\
(0,2241)\end{array}$ & $\begin{array}{l}-5,8835^{\star \star \star} \\
(1,5276)\end{array}$ & $\begin{array}{l}-5,9734^{* * *} \\
(2,1191)\end{array}$ & $\begin{array}{l}-6,1142^{\star \star \star} \\
(2,2596)\end{array}$ & $\begin{array}{l}-4,8464^{*} \\
(2,5743) \\
\end{array}$ & $\begin{array}{l}-4,9237^{*} \\
(2,9058)\end{array}$ \\
\hline Observações & 959 & 959 & 959 & 959 & 959 & 959 \\
\hline Pseudo $R^{2}$ & 0,0349 & 0,0998 & 0,1141 & 0,1318 & 0,1552 & 0,2230 \\
\hline Estatística $\chi^{2}$ & 14,44 & 69,00 & 71,76 & 95,67 & 111,29 & 174,58 \\
\hline
\end{tabular}

Erro-padrão entre parênteses. ${ }^{\star * \star} p<0,01 ;{ }^{\star *} p<0,05 ;{ }^{*} p<0,1$.

Fonte: Dados da pesquisa (2020).

O coeficiente da variável ICS mostrou-se estatisticamente significativo em todas as especificações estimadas, confirmando a hipótese de pesquisa. Os resultados alinham-se à literatura revisada, corroborando os resultados de Balsam et al. (2017) e de Hwang e Kim (2009). Evidencia-se que, mesmo com a inclusão das variáveis de controle, o ICS permaenece impactando de forma negativa a rotatividade involuntária do CEO. Ou seja, para as empresas da amostra, a presença de conexões sociais entre os membros do CA e o CEO diminui a probabilidade de rotatividade involuntária do CEO. A classificação estatística da especificação completa (6) pelo Teste de Hosmer-Lemeshow indicou que $92,28 \%$ dos eventos foram classificados adequadamente.

\section{Discussão dos Resultados}

Hwang e Kim (2009) indicam que a probabilidade de rotatividade involuntária do CEO diminui em 3,7 p.p. na presença de conexões sociais enquanto que no estudo de Balsam et al (2017) a redução é de 1,9 p.p.. Já o efeito do ICS na rotatividade involuntária neste estudo é de que a probabilidade de haver rotatividade involuntária de um CEO totalmente conectado diminui 19,03 p.p em relação a um CEO sem conexões sociais. Diferentemente dos estudos comparados, o ICS é um indicador contínuo. O efeito marginas estimado indica que probabilidade de rotatividade involuntária do CEO diminui em 2,34 p.p. para cada desvio-padrão do ICS.

A diferença de magnitude das probabilidades entre os estudos pode estar associada à utilização do ICS na presente pesquisa, visto que tanto Hwang e Kim (2009), quanto Balsam et al (2017) realizam as suas análises a partir de indicadores de conexão sociais isolados. O teste adicional realizado neste estudo e apresentado na Tabela 5 demonstra que os indicadores quando utilizados isoladamente não capturam adequadamente os efeitos das conexões sociais, mesmo na presença das variáveis que representam aspectos de GC. Neste sentido, uma análise agregada de dados através do ICS se mostra mais eficiente para 
demonstrar os efeitos das conexões sociais no monitoramento do CA, sendo esta uma contribuição deste artigo.

Os resultados evidenciam que a disposição individual ao monitoramente referida por Hermalin e Weisbach (1998), no mercado brasileiro, sofre maior influência das conexões sociais. Os resultados aparentemente não se relacionam com a concentração acionária característica do mercado brasileiro, visto que tal variável não apresentou significância para o modelo proposto, contrariando os achados de Vieira e Martins (2018). Por outro lado, a maior influência das conexões sociais no mercado nacional comparativamente ao mercado dos EUA pode estar associada à estrutura de controle das empresas. Isto porque a detenção do controle acionário por empresa estrangeira mostrou-se significativo, permitindo-se inferir que há maior monitoramento do CA ao desempenho do CEO em empresas cuja estrutura de controle não seja identificada com o Brasil.

Os resultados apresentados mantiveram-se significantes mesmo na presença de variáveis que representam outros aspectos de GC. Contrariando os manuais de boas práticas de GC, a independência formal do CA não se mostrou significativa para rotatividade involuntária do CEO, o que é, porém, consistente com os resultados de Vieira e Martins (2018). Estes achados oferecem suporte empírico para o mercado brasileiro ao que foi referido por Tung (2011), de que a independência formal excusivamente considerada não é suficiente para assegurar o monitoramento do CA.

Nenhuma das variáveis propostas quanto às características do CA apresentou significância para o modelo. Estes achados confrontam o estudo de Guo e Masulis (2015), desenvolvido com dados de empresas americanas, que indica que características estruturais do CA, como a presença de comitês de remuneração e $\mathrm{RH}$, afetam a rotatividade involuntária dos CEOs. Da mesma forma, os resultados contrariam, também, os achados de Vieira e Martins (2018), pois a dualidade do CEO não mostrou-se significativa. Os achados demonstram, portanto, que a análise das conexões sociais é pertinente no cenário brasileiro. Sob este enfoque, evidencia-se que as proxys usualmente utilizadas pela literatura para o monitoramento do CA em relação à rotatividade involuntária não capturam todos os seus determinantes, pois omitem as conexões sociais. Assim, o uso do ICS apresenta-se como alternativa mais eficiente para mensurar o monitoramento do CA.

Específicamente quanto às características do CEO, os resultados indicam que quanto mais velho 0 CEO, menor a chance de rotatividade involuntária o que é condizente com os estudo de Hoitash (2019).

Os resultados, portanto, oferecem evidências empíricas de que as conexões sociais influenciam o monitoramento do $C A$ quanto à rotatividade involuntária do CEO. Este fato pode decorrer de um maior envolvimento do CA nas decisões estratégicas da empresa (Adams \& Ferreira, 2007; Hoitash, 2011) em detrimento da função de monitoramento, dada a presença das conexões sociais. As evidências demonstram, também, que a utilização do ICS captura melhor o efeito das conexões sociais no monitoramento no contexto nacional do que indicadores isolados. Consiste, também, em uma melhor proxy de monitoramento do que as características estruturais do CA.

\section{Testes de robustez}

Adicionalmente foi testado o impacto do ICS na probabilidade da rotatividade classificada como voluntária. Os resultados são sintetizados na Tabela 4.

Tabela 4:

Resultados para rotatividade voluntária

\begin{tabular}{|c|c|c|c|c|c|c|}
\hline Variáveis & $\begin{array}{l}\text { (1) } \\
\text { ROTVOL }\end{array}$ & $\begin{array}{l}\text { (2) } \\
\text { ROTVOL }\end{array}$ & $\begin{array}{l}\text { (3) } \\
\text { ROTVOL }\end{array}$ & $\begin{array}{l}(4) \\
\text { ROTVOL }\end{array}$ & $\begin{array}{l}\text { (5) } \\
\text { ROTVOL }\end{array}$ & $\begin{array}{l}\text { (6) } \\
\text { ROTVOL }\end{array}$ \\
\hline$I C S$ & $\begin{array}{l}1,2820 \\
(0,8151)\end{array}$ & $\begin{array}{l}1,5687^{*} \\
(0,8946)\end{array}$ & $\begin{array}{l}2,1265^{\star \star} \\
(0,9674)\end{array}$ & $\begin{array}{l}2,3447^{\star \star} \\
(0,9845)\end{array}$ & $\begin{array}{l}2,5868^{\star \star \star} \\
(0,9938)\end{array}$ & $\begin{array}{l}2,6520^{\star *} \\
(1,0423)\end{array}$ \\
\hline Variáveis financeiras & Não & Sim & Sim & Sim & Sim & Sim \\
\hline Características do CA & Não & Não & Sim & Sim & Sim & Sim \\
\hline Variáveis de GC & Não & Não & Não & Sim & Sim & Sim \\
\hline $\begin{array}{l}\text { Características do } \\
\text { CEO }\end{array}$ & Não & Não & Não & Não & Sim & Sim \\
\hline D.ano & Não & Não & Não & Não & Não & Sim \\
\hline D.Setor & Não & Não & Não & Não & Não & Sim \\
\hline Constante & $\begin{array}{l}-2,2885^{\star \star \star} \\
(0,1922)\end{array}$ & $\begin{array}{l}-6,0068^{\star * *} \\
(1,3016)\end{array}$ & $\begin{array}{l}-7,4786^{\star \star \star} \\
(1,5824)\end{array}$ & $\begin{array}{l}-7,2086^{\star * *} \\
(1,6130)\end{array}$ & $\begin{array}{l}-8,7183^{\star * *} \\
(1,7520)\end{array}$ & $\begin{array}{l}-9,3347^{\star * *} \\
(2,0822)\end{array}$ \\
\hline Observações & 959 & 959 & 959 & 959 & 959 & 959 \\
\hline Pseudo $R^{2}$ & 0,0036 & 0,0328 & 0,0471 & 0,0528 & 0,1137 & 0,1320 \\
\hline Estatística $\chi^{2}$ & 2,47 & 43,56 & 49,47 & 95,67 & 102,52 & 131,16 \\
\hline
\end{tabular}

Erro-padrão entre parênteses. ${ }^{* \star *} p<0,01 ;{ }^{* \star} p<0,05 ;{ }^{*} p<0,1$

Fonte: Dados da pesquisa (2020).

Os resultados indicam que as chances de rotatividade voluntária aumentam na presença de conexões sociais entre os membros do CA e o CEO. Assim, o ICS exerce uma influência positiva na rotatividade 
voluntária, diferentemente do que ocorre na rotatividade involuntária quando o efeito é negativo. Este resultado é consistente com os estudos de Liu (2014), Renneboog e Zhao (2017) e Gao et al. (2017). Tais estudos indicam que na presença de conexões sociais os CEOs são mais propensos a deixarem as empresas por terem maior poder de barganha junto ao CA dificultando uma negociação que tenha por objetivo 0 benefício dos proprietários em detrimento dos interesses do CEO.

Adicionalmente, a exemplo da literatura precedende, a qual mensura a conexão social entre o CEO e os membros do CA através de indicadores isolados, testaram-se os efeitos dos indicadores que compuseram o ICS na rotatividade involuntária do CEO. Os resultados das seis especificações estão sintetizados na Tabela 5.

Tabela 5:

Resultados para rotatividade involuntária a partir dos indicadores

\begin{tabular}{|c|c|c|c|c|c|c|}
\hline Variáveis & $\begin{array}{l}\text { (1) } \\
\text { ROTINV }\end{array}$ & $\begin{array}{l}\text { (2) } \\
\text { ROTINV }\end{array}$ & $\begin{array}{l}\text { (3) } \\
\text { ROTINV }\end{array}$ & $\begin{array}{l}(4) \\
\text { ROTINV }\end{array}$ & $\begin{array}{l}\text { (5) } \\
\text { ROTINV }\end{array}$ & $\begin{array}{l}(6) \\
\text { ROTINV }\end{array}$ \\
\hline IES & $\begin{array}{l}-4.313^{* * *} \\
(1.53)\end{array}$ & $\begin{array}{l}-3.898^{\star *} \\
(1.58)\end{array}$ & $\begin{array}{l}-3.421^{\star *} \\
(1.53)\end{array}$ & $\begin{array}{l}-3.201^{* *} \\
(1.57)\end{array}$ & $\begin{array}{l}-3.086^{\star} \\
(1.63)\end{array}$ & $\begin{array}{l}-2.887^{*} \\
(1.63)\end{array}$ \\
\hline Diretoria Executiva & $\begin{array}{l}0.851 \\
(0.91)\end{array}$ & $\begin{array}{l}1.319 \\
(1.02)\end{array}$ & $\begin{array}{l}1.104 \\
(0.97)\end{array}$ & $\begin{array}{l}1.077 \\
(0.98)\end{array}$ & $\begin{array}{l}1.030 \\
(0.97)\end{array}$ & $\begin{array}{l}0.753 \\
(0.99)\end{array}$ \\
\hline Experiência Profissional & $\begin{array}{l}-0.589 \\
(0.59)\end{array}$ & $\begin{array}{l}-0.623 \\
(0.66)\end{array}$ & $\begin{array}{l}-0.722 \\
(0.68)\end{array}$ & $\begin{array}{l}-0.900 \\
(0.66)\end{array}$ & $\begin{array}{l}-0.574 \\
(0.68)\end{array}$ & $\begin{array}{l}-0.434 \\
(0.76)\end{array}$ \\
\hline Experiência em $C A$ & $\begin{array}{l}-0.748^{* *} \\
(0.35)\end{array}$ & $\begin{array}{l}-0.641^{*} \\
(0.37)\end{array}$ & $\begin{array}{l}-0.616^{\star} \\
(0.36)\end{array}$ & $\begin{array}{l}-0.499 \\
(0.35)\end{array}$ & $\begin{array}{l}-0.448 \\
(0.35)\end{array}$ & $\begin{array}{l}-0.632^{*} \\
(0.36)\end{array}$ \\
\hline Conexão Familiar & $\begin{array}{l}-4.208^{\star *} \\
(1.91)\end{array}$ & $\begin{array}{l}-4.107^{\star *} \\
(1.85)\end{array}$ & $\begin{array}{l}-3.996^{\star *} \\
(1.93)\end{array}$ & $\begin{array}{l}-3.410^{*} \\
(1.79)\end{array}$ & $\begin{array}{l}-2.937 \\
(1.94)\end{array}$ & $\begin{array}{l}-2.873 \\
(2.16)\end{array}$ \\
\hline Variáveis financeiras & Não & Sim & Sim & Sim & Sim & Sim \\
\hline Características do CA & Não & Não & Sim & Sim & Sim & Sim \\
\hline Variáveis de GC & Não & Não & Não & Sim & Sim & Sim \\
\hline Características do CEO & Não & Não & Não & Não & Sim & Sim \\
\hline D.ano & Não & Não & Não & Não & Não & Sim \\
\hline D.Setor & Não & Não & Não & Não & Não & Sim \\
\hline Constante & $\begin{array}{l}-1.743 \\
(0.24)\end{array}$ & $\begin{array}{l}-5.062 \\
(1.73)\end{array}$ & $\begin{array}{l}-4.567 \\
(2.29)\end{array}$ & $\begin{array}{l}-4.722 \\
(2.50)\end{array}$ & $\begin{array}{l}-3.627 \\
(2.76)\end{array}$ & $\begin{array}{l}-3.805 \\
(3.08)\end{array}$ \\
\hline Observações & 959 & 959 & 959 & 959 & 959 & 959 \\
\hline Pseudo $R^{2}$ & 0,0660 & 0,1268 & 0,1345 & 0,1479 & 0,1678 & 0,2324 \\
\hline Estatística $\chi^{2}$ & 17,70 & 65,08 & 69,68 & 87,69 & 106,43 & 180,20 \\
\hline
\end{tabular}

Erro-padrão entre parênteses. ${ }^{\star \star *} p<0,01 ;{ }^{* \star} p<0,05 ;{ }^{*} p<0,1$

Fonte: Dados da pesquisa (2020).

Os dados indicam que nenhum dos indicadores que compõem o ICS quando analisados isoladamente é significativo à $5 \%$ de significância na especificação completa (6). Observa-se que os indicadores, ao contrário do que ocorre com o ICS, perdem significância com a agregação das variáveis de controle ao modelo. Esta evidência demonstra que os indicadores quando analisados isoladamente não capturam o efeito do fenômeno observado tornando relevante e pertinente a utilização de dados agregados através do índice proposto no cenário brasileiro.

\section{Considerações Finais}

O objetivo do presente estudo foi mensurar o impacto das conexões sociais existentes entre os membros do CA e o CEO na rotatividade involuntária do CEO nas empresas listadas na $[B]^{3}$ mediante um Índice de Conexão Social (ICS). Neste sentido, entende-se que a pesquisa preenche uma importante lacuna, pois fornece dados acerca da atuação dos mecanismos de controle de GC na presença de conexões sociais em um ambiente de concentração acionária. Os resultados apontam que a absoluta maioria das empresas $(93,22 \%)$ apresenta algum grau de conexão entre o CEO e os membros do CA. Este resutado é superior ao encontrado por estudos similares em outros países podendo indicar que a alta conectividade entre CEO e CA é uma característica própria do mercado brasileiro (Balsam et al., 2017; Hwang \& Kim, 2009).

Os resultados confirmam que na presença de conexões sociais, há menor probabilidade de rotatividade involuntária do CEO e que esta não relaciona-se com a concentração acionária ou com características estruturais do CA contrariando estudos anteriores (Vieira \& Martins, 2018; Guo \& Masulis, 2015).

Os resultados contribuem, também, para o debate acerca das caracterísiticas não observáveis do CA capazes de influenciar no seu monitoramento. Isto porque a influência das conexões sociais na rotatividade involuntária do CEO manteve-se mesmo na presença de proxys para boas práticas de governança. A pesquisa agrega, portanto, à literatura de governança ao fornecer subsídios para análise das práticas de GC não relacionadas a questões estruturais do CA. Neste sentido, o ICS se apresenta como uma proxy mais eficiente para o monitoramento do CA.

Da mesma forma, os achados e, essencialmente o ICS proposto, fornecem aos stakeholders uma nova perspectiva de análise quanto às práticas de Governança Corporativa. O teste de robustez evidenciou 
que os indicadores usualmente relacionados pela literatura para conexão social testados de modo isolado não mostraram-se significativos. Este resultado sinaliza que a análise agregada de dados através do índice proposto se mostra relevante para capturar os efeitos das interações sociais entre os membros do CA e o CEO no mercado brasileiro, podendo ser usadas para outras finalidades ou ações de monitoramento de responsabilidade do CA.

Destaca-se que a pesquisa apresenta limitações quanto à classificação da rotatividade involuntária, pois foram adotados critérios indicados na literatura prévia. Limita-se, ainda, quanto ao ICS, dado que foram selecionados indicadores baseados na literatura revisada, bem como se limita pelo emprego de dados oriundos do Formulário de Referência na construção das variáveis os quais podem conter algum erro ou incorreção. Entretanto, entende-se que tais limitações não retiram a relevância da pesqusia para a discussão do monitoramento do CEO pelo CA na presença de conexões sociais.

Sugere-se para estudos futuros a aplicação de medidas alternativas de rotatividade que contemplem as empresas não listadas na $[\mathrm{B}]^{3}$. Indica-se, também, a agregação de outros indicadores peculiares ao cenário nacional, tal como a conectividade via relação política.

\section{Referências}

Adams, R. B., \& Ferreira, D. (2007). A theory of friendly boards. The Journal of Finance, 62(1), 217-250. https://doi.org/10.1111/j.1540-6261.2007.01206.x

Alexandre, N. M. C., \& Coluci, M. Z. O. (2011). Validade de conteúdo nos processos de construção e adaptação de instrumentos de medidas. Ciência \& Saúde Coletiva, 16, 3061-3068. http://dx.doi.org/10.1590/S1413-81232011000800006

Balsam, S., Kwack, S. Y., \& Lee, J. Y. (2017). Network connections, CEO compensation and involuntary turnover: The impact of a friend of a friend. Journal of Corporate Finance, 45, 220-244. https://doi.org/10.1016/i.jcorpfin.2017.05.001

Beiner, S., Drobetz, W., Schmid, M. M., \& Zimmermann, H. (2006). An integrated framework of corporate governance and firm valuation. European Financial Management, 12(2), 249-283.

https://doi.org/10.1111/j.1354-7798.2006.00318.x

Black, B., Carvalho, A. G., Khanna, V., Kim, W., \& Yurtoglu, B. (2017). Corporate governance indices and construct validity. Corporate Governance: An International Review, 25(6), 397-410. https://doi.org/10.1111/corg.12215

Brasil, Bolsa, Balcão [B3]. (2018). Regulamento do novo mercado. Disponível em http://www.b3.com.br/pt br/regulacao/estrutura-normativa/listagem/

Brookman, J., \& Thistle, P. D. (2009). CEO tenure, the risk of termination and firm value. Journal of Corporate finance, 15(3), 331-344. https://doi.org/10.1016/j.jcorpfin.2009.01.002

Brown, L. D., \& Caylor, M. L. (2004). Corporate governance and firm performance. Available at SSRN 586423. https://dx.doi.org/10.2139/ssrn.586423

Bushman, R., Dai, Z., \& Wang, X. (2010). Risk and CEO turnover. Journal of Financial Economics, 96(3), 381-398. https://doi.org/10.1016/j.jfineco.2010.03.001

Cadbury, A. (1992). Report of the Committee on the financial aspects of Corporate Governance, Gee \& Co. Ltd., London, United Kingdom

Campbell, S. M., Braspenning, J. A., Hutchinson, A., \& Marshall, M. (2002). Research methods used in developing and applying quality indicators in primary care. Quality and Safety in Health Care, 11(4), 358364. http://dx.doi.org/10.1136/ahc. 11.4 .358

Campbell, T. C., Gallmeyer, M., Johnson, S. A., Rutherford, J., \& Stanley, B. W. (2011). CEO optimism and forced turnover. Journal of Financial Economics, 101(3), 695-712.

https://doi.org/10.1016/j.jfineco.2011.03.004

Céspedes, J., González, M., \& Molina, C. A. (2010). Ownership and capital structure in Latin America. Journal of business research, 63(3), 248-254. https://doi.org/10.1016/j.jbusres.2009.03.010

Chahine, S., \& Goergen, M. (2013). The effects of management-board ties on IPO performance. Journal of 
Corporate Finance, 21, 153-179. https://doi.org/10.1016/j.jcorpfin.2013.02.001

Chahine, S., \& Goergen, M. (2014). Top management ties with board members: How they affect payperformance sensitivity and IPO performance. Journal of Corporate Finance, 27, 99-115. https://doi.org/10.1016/j.jcorpfin.2014.04.007

Cohen, L., Frazzini, A., \& Malloy, C. (2010). Sell-side school ties. The Journal of Finance, 65(4), 1409-1437. https://doi.org/10.1111/j.1540-6261.2010.01574.x

Coles, J. L., Daniel, N. D., \& Naveen, L. (2014). Co-opted boards. The Review of Financial Studies, 27(6), 1751-1796. https://doi.org/10.1093/rfs/hhu011

Cooper, D. R., \& Schindler, P. S. (2016). Métodos de Pesquisa em Administração-12ª edição. McGraw Hill Brasil.

Crisóstomo, V. L., de Freitas Brandão, I., \& López-Iturriaga, F. J. (2020). Large shareholders' power and the quality of corporate governance: An analysis of Brazilian firms. Research in International Business and Finance, 51, 101076. https://doi.org/10.1016/j.ribaf.2019.101076

Fracassi, C., \& Tate, G. (2012). External networking and internal firm governance. The Journal of Finance, 67(1), 153-194. https://doi.org/10.1111/j.1540-6261.2011.01706.x

Gao, H., Harford, J., \& Li, K. (2017). CEO turnover-performance sensitivity in private firms. Journal of Financial and Quantitative Analysis, 52(2), 583-611. https://doi.org/10.1017/S0022109017000126

Grant, J. S., \& Davis, L. L. (1997). Selection and use of content experts for instrument development. Research in nursing \& health, 20(3), 269-274. https://doi.org/10.1002/(SICl)1098240X(199706)20:3\%3C269::AID-NUR9\%3E3.0.CO;2-G

Gujarati, D. N., \& Porter, D. C. (2011). Econometria básica-5. Amgh Editora.

Guo, L., \& Masulis, R. W. (2015). Board structure and monitoring: New evidence from CEO turnovers. The Review of Financial Studies, 28(10), 2770-2811. https://doi.org/10.1093/rfs/hhv038

Harris, M., \& Raviv, A. (2008). A theory of board control and size. The Review of Financial Studies, 21(4), 1797-1832. https://doi.org/10.1093/rfs/hhl030

Hermalin, B. E., \& Weisbach, M. S. (1998). Endogenously chosen boards of directors and their monitoring of the CEO. American Economic Review, 96-118.

Hoitash, U. (2011). Should independent board members with social ties to management disqualify themselves from serving on the board?. Journal of Business Ethics, 99(3), 399-423.

https://doi.org/10.1007/s10551-010-0660-5

Hoitash, U., \& Mkrtchyan, A. (2019). Directors' ties to non-CEO executives: Information advantage or entrenchment?. Available at SSRN 3372471. https://doi.org/10.2139/ssrn.3372471

Hsu, C. C., \& Sandford, B. A. (2007). The Delphi technique: making sense of consensus. Practical Assessment, Research, and Evaluation, 12(1), 10. https://doi.org/10.7275/pdz9-th90

Hu, J., \& Kim, J. B. (2019). The relative usefulness of cash flows versus accrual earnings for CEO turnover decisions across countries: The role of investor protection. Journal of International Accounting, Auditing and Taxation, 34, 91-107. https://doi.org/10.1016/j.intaccaudtax.2019.02.005

Hwang, B. H., \& Kim, S. (2009). It pays to have friends. Journal of financial economics, 93(1), 138-158. https://doi.org/10.1016/j.jfineco.2008.07.005

IBGC - Instituto Brasileiro de Governança Corporativa. (2015). Código das melhores práticas de governança corporativa.

Interagentes, G. (2016). Código brasileiro de governança corporativa. Instituto Brasileiro de Governança Corporativa-IBGC. 
Ishii, J., \& Xuan, Y. (2014). Acquirer-target social ties and merger outcomes. Journal of Financial Economics, 112(3), 344-363. https://doi.org/10.1016/j.jfineco.2014.02.007

Jensen, M. C. (1993). The modern industrial revolution, exit, and the failure of internal control systems. The Journal of Finance, 48(3), 831-880. https://doi.org/10.1111/j.1540-6261.1993.tb04022.x

Jones, J., \& Hunter, D. (1995). Qualitative research: consensus methods for medical and health services research. Bmj, 311(7001), 376-380. https://doi.org/10.1136/bmi.311.7001.376

Kang, J. K., Liu, W. L., Low, A., \& Zhang, L. (2018). Friendly boards and innovation. Journal of Empirical Finance, 45, 1-25. https://doi.org/10.1016/.j.jempfin.2017.09.007

Kaplan, S. N., \& Minton, B. A. (2012). How has CEO turnover changed? International Review of Finance, 12(1), 57-87. https://doi.org/10.1111/i.1468-2443.2011.01135.x

Khedmati, M., Sualihu, M. A., \& Yawson, A. (2020). CEO-director ties and labor investment efficiency. Journal of Corporate Finance, 65, 101492. https://doi.org/10.1016/.j.jcorpfin.2019.101492

Knyazeva, A., Knyazeva, D., \& Masulis, R. W. (2013). The supply of corporate directors and board independence. The Review of Financial Studies, 26(6), 1561-1605. https://doi.org/10.1093/rfs/hht020

Krishnan, G. V., Raman, K. K., Yang, K., \& Yu, W. (2011). CFO/CEO-board social ties, Sarbanes-Oxley, and earnings management. Accounting Horizons, 25(3), 537-557. https://doi.org/10.2308/acch-50028

La Porta, R., Lopez-de-Silanes, F., \& Shleifer, A. (1999). Corporate ownership around the world. The Journal of Finance, 54(2), 471-517. https://doi.org/10.1111/0022-1082.00115

Silva, A. L. P. D., Lana, J., \& Marcon, R. (2018). Agreeing and impacting: The effect of the shareholders' agreement on firms' market value. BBR. Brazilian Business Review, 15(1), 88-104.

https://doi.org/10.15728/bbr.2018.15.1.6

Liu, Y. (2014). Outside options and CEO turnover: The network effect. Journal of Corporate Finance, 28, 201-217. https://doi.org/10.1016/j.jcorpfin.2014.03.004

Lynn, M. R. (1986). Determination and quantification of content validity. Nursing Research, 35(6), 382-385. https://doi.org/10.1097/00006199-198611000-00017

Martins, G. (2006). Sobre confiabillidade e validade. Revista Brasileira de Gestão de Negócios-RBGN, 8(20), 1-12. https://doi.org/10.7819/rbgn.v8i20.51

McGuinness, P. B. (2018). IPO firm performance and its link with board officer gender, family-ties and other demographics. Journal of Business Ethics, 152(2), 499-521. https://doi.org/10.1007/s10551-016-3295-3

McPherson, M., Smith-Lovin, L., \& Cook, J. M. (2001). Birds of a feather: Homophily in social networks. Annual Review of Sociology, 27(1), 415-444. https://doi.org/10.1146/annurev.soc.27.1.415

Nguyen, B. D. (2012). Does the Rolodex matter? Corporate elite's small world and the effectiveness of boards of directors. Management Science, 58(2), 236-252. https://doi.org/10.1287/mnsc.1110.1457

OECD - Organización para la Cooperación y el Desarrollo. (2016). Directrices de la OCDE sobre el Gobierno Corporativo de las Empresas Públicas.

Parrino, R. (1997). CEO turnover and outside succession a cross-sectional analysis. Journal of financial Economics, 46(2), 165-197.

Perlin, M., Kirch, G., \& Vancin, D. (2018). Accessing financial reports and corporate events with GetDFPData. Available at SSRN 3128252. https://dx.doi.org/10.2139/ssrn.3128252

Polit, D. F., \& Beck, C. T. (2006). The content validity index: are you sure you know what's being reported? Critique and recommendations. Research in Nursing \& Health, 29(5), 489-497.

https://doi.org/10.1002/nur.20147

Renneboog, L., \& Zhao, Y. (2020). Director networks, turnover, and appointments. European Financial 
Management, 26(1), 44-76. https://doi.org/10.1111/eufm.12213

Schmidt, B. (2015). Costs and benefits of friendly boards during mergers and acquisitions. Journal of Financial Economics, 117(2), 424-447. https://doi.org/10.1016/j.jfineco.2015.02.007

Sutton, C., Veliyath, R., Pieper, T. M., Hair Jr, J. F., \& Caylor, M. (2018). Secondary agency conflicts: A synthesis and proposed measurement model. Long Range Planning, 51(5), 720-735.

https://doi.org/10.1016/i.lrp.2017.12.004

Tung, F. (2011). The Puzzle of Independent Directors: New Learning. Boston University Law Review, 91(3), 1175-1190.

Vieira, C. A., \& Martins, O. (2018). Influência da estrutura do conselho de administração e do controle corporativo no turnover do CEO das empresas abertas no Brasil. Revista Contemporânea de Contabilidade, 15(34), 181-201. https://doi.org/10.5007/2175-8069.2018v15n34p181

Westphal, J. D. (1999). Collaboration in the boardroom: Behavioral and performance consequences of CEOboard social ties. Academy of Management Journal, 42(1), 7-24. https://doi.org/10.5465/256871

Young, M. N., Peng, M. W., Ahlstrom, D., Bruton, G. D., \& Jiang, Y. (2008). Corporate governance in emerging economies: A review of the principal-principal perspective. Journal of Management Studies, 45(1), 196-220. https://doi.org/10.1111/j.1467-6486.2007.00752.x

\section{NOTAS}

\section{AGRADECIMENTOS}

Não se aplica

\section{CONTRIBUIÇÃO DE AUTORIA}

Concepção e elaboração do manuscrito: L.G. Locatelli, C.M. Costa

Coleta de dados: F.M. Ramos, L.G. Locatelli

Análise de dados: L.G. Locatelli, C. M. Costa

Discussão dos resultados: L.G. Locatelli, F.M. Ramos, C.M. Costa

Revisão e aprovação: L.G. Locatelli, F.M. Ramos, C.M. Costa

\section{CONJUNTO DE DADOS DE PESQUISA}

O conjunto de dados que dá suporte aos resultados deste estudo não está disponível publicamente.

\section{FINANCIAMENTO}

O presente trabalho foi realizado com apoio da Coordenação de Aperfeiçoamento de Pessoal de Nível Superior - Brasil (CAPES) - Código de Financiamento 001.

O presente trabalho foi realizado com apoio do Conselho Nacional de Desenvolvimento Científico e Tecnológico - CNPq

- Brasil, através do Processo 438732/2018-9.

\section{CONSENTIMENTO DE USO DE IMAGEM}

Não se aplica.

\section{APROVAÇÃO DE COMITÊ DE ÉTICA EM PESQUISA}

Não se aplica.

\section{CONFLITO DE INTERESSES}

Não se aplica.

\section{LICENÇA DE USO}

Os Direitos Autorais para artigos publicados neste periódico são do autor, com direitos de primeira publicação para a Revista. Em virtude de aparecerem nesta Revista de acesso público, os artigos são de uso gratuito, com atribuições próprias, em aplicações educacionais, de exercício profissional e para gestão pública. A Revista adotou a licença Creative Commons Atribuição 4.0 Internacional - CC BY NC ND. Esta licença permite acessar, baixar (download), copiar, imprimir, compartilhar, reutilizar e distribuir os artigos desde que com a citação da fonte, atribuindo os devidos créditos de autoria. Nesses casos, nenhuma permissão é necessária por parte dos autores ou dos editores. Autores têm autorização para assumir contratos adicionais separadamente, para distribuição não-exclusiva da versão do trabalho publicada nesta revista (ex.: publicar em repositório institucional ou um capítulo de livro). 


\section{PUBLISHER}

Universidade Federal de Santa Catarina. Curso de Ciências Contábeis e Programa de Pós-graduação em Contabilidade. Publicação no Portal de Periódicos UFSC. As ideias expressadas neste artigo são de responsabilidade de seus autores, não representando, necessariamente, a opinião dos editores ou da universidade.

\section{EDITORES}

Carlos Eduardo Facin Lavarda e Suliani Rover

\section{HISTÓRICO}

Recebido em: 31/07/2020 - Revisado por pares em: 18/02/2021 - Reformulado em: 30/04/2021 Recomendado para publicação em: 07/05/2021 - Publicado em: 30/06/2020

* Peprint do artigo apresentado no Congresso ANPCONT, 2020 